\title{
Nomadisme (Saharien en Afrique du Nord dans l'antiquité)
}

Pol Trousset

\section{(2) OpenEdition}

1 Journals

Édition électronique

URL : https://journals.openedition.org/encyclopedieberbere/2750

DOI : 10.4000/encyclopedieberbere. 2750

ISSN : 2262-7197

Éditeur

Peeters Publishers

\section{Édition imprimée}

Date de publication : 5 octobre 2012

Pagination : 5578-5589

ISBN : 978-90-429-2718-6

ISSN : 1015-7344

Référence électronique

Pol Trousset, « Nomadisme (Saharien en Afrique du Nord dans l'antiquité) », Encyclopédie berbère [En ligne], 34 | 2012, document N64, mis en ligne le 15 décembre 2020, consulté le 17 février 2022. URL: http://journals.openedition.org/encyclopedieberbere/2750 ; DOI : https://doi.org/10.4000/ encyclopedieberbere. 2750

Ce document a été généré automatiquement le 17 février 2022.

(c) Tous droits réservés 


\title{
Nomadisme (Saharien en Afrique du Nord dans l'antiquité)
}

\author{
Pol Trousset
}

\section{Les nomades sahariens dans l'historiographie antique : une image négative}

1 Dans sa présentation de l'Afrique au début de la guerre de Jugurtha, l'historien Salluste donne du pays où il avait exercé lui-même de hautes fonctions administratives, une vue cavalière qui s'ordonne à la fois d'est en ouest et du nord au sud vers l'intérieur du continent, à partir des anciennes possessions puniques et du royaume de Numidie : «Les autres régions jusqu'à la Maurétanie sont occupées par les Numides; les plus près de l'Espagne sont les Maures. Au-dessus (c'est-à-dire au-delà) de la Numidie se trouvent, dit-on, les Gétules, qui vivent les uns dans des huttes, les autres plus barbares en nomades ; derrières eux les Éthiopiens ; enfin des régions embrasées par les ardeurs du soleil ».

2 Dans ces profondeurs de la Libye intérieure, d'autres peuples, les Nasamons* et les Garamantes* avaient déjà été répertoriés au temps d'Hérodote. De la steppe ou des hautes plaines jusqu'au Grand Désert, plusieurs ensembles de populations apparaissent donc ainsi en dégradé. On retiendra les deux exemples les plus représentatifs de ces nomades pour tenter d'en dégager l'image dominante qu'en avaient donnée les auteurs anciens.

\section{Les Gétules*}

3 Il s'agit d'un terme vague dont on sait seulement qu'il s'appliquait non à une confédération de tribus comme celle des Musulames*, mais à l'un des ensembles de peuples qui avec ceux des Maures* et des Numides* habitaient une large zone du Maghreb actuel (fig. 1). Leur localisation est également incertaine car on en trouve du sud marocain (les Autololes*) jusqu'au pays des Syrtes avec les Cinithii* et les Maces*, 
comme si, selon la formule de G. Camps, «à partir d'une certaine latitude les Libyens prenaient automatiquement ce nom ». Mais on peut placer la limite septentrionale de la Gétulie à Madaure si l'on se réfère au témoignage d'Apulée natif de cette ville et qui se disait mi-Numide et mi-Gétule (Apol, 24,1). Ainsi pouvait-elle englober le pays un peu plus sédentarisé des Musulames qui était en relation avec les rois numides et où l'urbanisation paraît avoir des racines anciennes autour de villages fortifiés (castella). Vers le sud pour Pline l'Ancien, la limite de la Gétulie était sur le fleuve Nigris (Oued Djedi) au sud de l'Atlas saharien et plus généralement dans les oasis présahariennes où se concentraient les Éthiopiens mélanodermes. En définitive, c'est dans un sens avant tout politique qu'on pourrait le mieux les définir : S. Gsell, suivi en cela par J. Desanges, estimait qu'il s'agissait de peuples restés en marge des royaumes maures ou numides et rentrés plus tardivement dans la mouvance de Rome. Leur structure tribale qui les faisait désigner par les auteurs anciens sous le terme de gentes* (ou de civitas) les aurait rendus longtemps réfractaires à la notion d'Etat ou de cité à la romaine. Leur marginalité à la fois géographique et anthropologique aurait préservé pendant plusieurs siècles leur identité. En contact difficile avec Rome à l'époque de Juba II, ils ne seront pas toujours hostiles au pouvoir romain à qui ils fourniront même des soldats dès l'époque de Marius; ils n'en resteront pas moins considérés pendant longtemps comme fuyants et insaisissables, des Gétules installés dans l'errance : des (b) vagi Getuli qui sur la Table de Peutinger, désignent les Maces. Comme le soulignait G. Camps, c'est donc bien leur genre de vie marqué par un nomadisme plus ou moins affirmé qui, associé à la structure tribale de leur société, serait le fondement de cette identité persistante. Tandis que Maures et Numides habitaient le Tell où l'agriculture était possible et parfois prédominante, les divers peuples Gétules auraient été très majoritairement des nomades - ou des semi-nomades saisonniers - jusqu'à l'occupation militaire romaine des régions allant des Hautes-Plaines à la steppe et au pré-désert. À vrai dire, dans un vaste ensemble de régions hétérogènes qui prenait en écharpe l'Afrique du Nord des rives de l'Atlantique à celles des Syrtes, la distinction des diverses populations par leur genre de vie et leurs domaines géographiques respectifs était loin d'être évidente comme le suggère au passage le jeu de mots Nomades/Numides repris par Strabon (II, 5, 33) et qui révèle combien peut être sujet à caution l'emploi, à partir de l'historiographie antique, du concept de nomadisme.

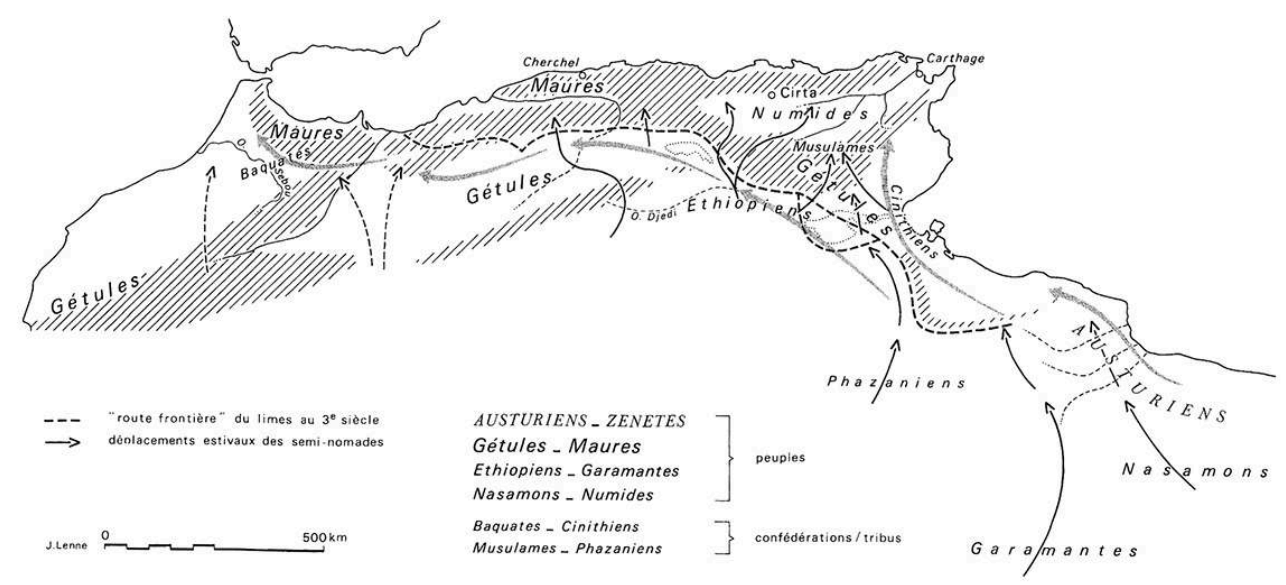

Fig. 1. CARTE des POPULATIONS ET deS MOUVEMENTS NOMADES DANS L'ANTIQUITÉ 


\section{Les Garamantes*}

$4 \quad$ Nul autre peuple que celui de ces grands caravaniers retranchés dans les profondeurs "éthiopiennes» de l'Afrique n'a inspiré aux auteurs anciens autant de clichés fantasmagoriques et ceci dès l'époque d'Hérodote mais plus tard encore jusque dans l'antiquité tardive où Orose par exemple les situent dans une géographie mythique, sur les rives d'un océan austral « Éthiopique ». La distance qui les séparait des frontières de l'Empire pourrait expliquer la persistance des stéréotypes les concernant. Malgré le halo poétique qui enveloppe son nom, ce peuple présente pourtant une réelle consistance historique et on s'accorde à penser qu'il s'était organisé en une manière d'Etat dont la stabilité était fondée sur le double contrôle des routes sahariennes et des oasis du Fezzan. Leur aire de circulation s'étendait vers le nord et le nord-ouest en Tripolitaine par Ghadamès (Cidamus) où ils étaient en relation avec les Gétules et pouvaient menacer les villes côtières comme en 70 de notre ère ; vers l'est, en arrière de la grande Syrte, jusqu'à Zéla, en direction d'Augila où se trouvaient les Nasamons. Sans doute parcouraient-ils le désert au sud jusqu'au Tassili des Ajjer. Mais on ignore où il convient de placer le mystérieux pays d'Agisymba où se voyaient des rhinocéros et où une expédition romaine aurait accompagné le chef des Garamantes en guerre contre les Éthiopiens. Le centre de la puissance garamantique était au Fezzan, notamment dans la vallée du Wadi el-Agial au sud de la Hamada el-Homra où se trouvait la capitale Garama* (Djerma) bien connue depuis les travaux des équipes britanniques. Les Garamantes étaient un peuple expert en hydraulique puisqu'ils connaissaient déjà la foggara. L'information archéologique concernant ce peuple est donc assez substantielle pour qu'il soit intéressant de la mettre en parallèle avec celle fournie par les textes.

5 La première description est celle d'Hérodote (IV, 183) qui cite les Garamantes parmi d'autres peuples, les Ammoniens et les Nasamons échelonnés d'est en ouest à l'intérieur de la Libye, à une distance de dix jours d'Augila et « où il y a un tertre de sel avec de l'eau et beaucoup de dattiers... c'est un peuple fort nombreux ; ils apportent de la terre sur le sel et, cela fait, ensemencent. La route la plus directe (vers le littoral)... aboutit chez les Lotophages; de là à leur pays, il y a trente journées de chemin ». Vient ensuite cette notation étrange, mais essentielle peut-être, pour caractériser ce peuple : «c'est chez eux que se trouvent aussi les bœufs qui paissent à reculons. Voici pourquoi ces bœufs paissent ainsi : ils ont les cornes inclinées en avant; cela les oblige à paître en reculant; ils ne peuvent en effet paittre en avançant, parce que leurs cornes s'enfonceraient devant eux dans la terre... ces Garamantes donnent la chasse sur leurs chars à quatre chevaux aux Troglodytes Ethiopiens». On retrouve bien dans cette description certains éléments de réalité géographique ou ethnographique; l'échelonnement des oasis, la distance à la côte, les chars garamantiques. Des chars sont bien figurés sur les fresques du Tassili, mais on admet aujourd'hui que ces attelages sophistiques avaient surtout un caractère de parade. Quant à la rétrogression des bœufs garamantiques, Gabriel Camps l'expliquait avec beaucoup d'humour par quelque récit de «cabaret » à des citadins grecs ou libyens du littoral, d'un caravanier du grand désert inspiré par les fresques peintes par ses lointains aïeux. «Le bruit en serait arrivé jusqu'à Hérodote qui le consigna sur ses tablettes». Il pourrait y avoir malgré l'interprétation laborieuse qu'en donne ce dernier, une portée symbolique bien plus large si on le met en parallèle avec un discours mythique déjà développé par Homère : il s'agit des bœufs d'Apollon dérobés par le jeune Hermès qui les fait paitre à reculons pour tromper ses poursuivants. C'est ce qu'on peut lire dans l'hymne à Hermès (I, 
$75-80)$ : « Il les poussait devant lui - piste illusoire ! - à travers le terrain sablonneux, en retournant leurs traces; il n'oubliait pas ses talents de ruse quand il renversa les empreintes des sabots - les premiers en arrière, ceux de derrière en avant - tandis qu'il marchait lui-même en sens contraire ». Or, si Hermès est le dieu des voyageurs, il est aussi celui des voleurs; dans l'hymne homérique il est celui qui inverse les signes de reconnaissance: c'est un brouilleur de pistes. En plaçant l'évocation du peuple des Garamantes sous le signe inquiétant de la figure ambiguë d'Hermès, Hérodote s'inscrit dans la tradition géographique selon laquelle ceux-ci appartenaient au monde insaisissable et retors des trafiquants et des brigands. C'est de ce trait que restera marqué, dans toute l'historiographie antique, le stéréotype du nomade. Ainsi, la description de ce peuple par Pline l'Ancien (H.N., V, 14) continue de véhiculer les mêmes clichés : on reconnaît transposée en termes réalistes, l'idée exprimée dans le passage d'Hérodote par le mythe des bœufs d'Apollon. "Démêler l'itinéraire conduisant chez les Garamantes fut impossible jusqu à nos jours, les brigands (latrones) de cette nation recouvrant de sable leurs puits qui n'ont pas à être creusés profondément, si l'on est servi par la connaissance des lieux». Or cinq siècles environ séparent le temps de Pline de celui d'Hérodote, les Romains ont eu un contact direct avec ce peuple lointain qui a envoyé des ambassades à Rome après avoir été mêlé aux guerres Gétules (Tacite, Ann., IV, 26) ; plusieurs expéditions punitives ou de reconnaissance ont été envoyées chez les Garamantes, dont une des dernières est celle de Valerius Festus à laquelle il est fait allusion dans ce passage de Pline. Après quoi, à partir des Flaviens, les relations semblent s'être normalisées pour longtemps le pays garamante devenant une sorte de protectorat romain voué a un commerce caravanier sur de longues distances.

6 La connaissance géographique ayant beaucoup progressé du fait de la conquête romaine, les préjugés concernant les peuples sahariens comme tous les autres nomades errant aux lisières extrêmes du monde romain et de ce fait, à l'horizon de l'humanité, n'en demeuraient pas moins vivaces : leur vie entière comme le dira plus tard Ammien Marcellin à propos des Saracènes du désert de Syrie, "est semper in fuga ». Elle ressemble « à une fuite, à une course précipitée qui ne mène nulle part : ils multiplient sans fin leur circuit tourbillonnant d'illusion en illusion, êtres inconsistants dans leur effervescence stérile » (cf. Dauge, op. cit., p. 625). Ainsi ces nomades « vêtus de peaux de chèvres" comme le notait Diodore au sujet des Libyens pillards de l'intérieur, représentaient-ils pour les Romains une quintessence de la barbarie et pour Aristote déjà (Politique, 1250 a, 40-41), une sorte de degré zéro de l'humanité.

\section{Rome et les nomades : contacts et conflits}

7 Il faut, pour comprendre dans sa logique le processus de l'occupation romaine, revenir à la situation résultant de l'annexion, à l'époque augustéenne, des vastes territoires relevant auparavant des princes numides et incorporés désormais dans la province de l'Africa Proconsularis. Sur les marges occidentales et méridionales de cette dernière, Rome se trouvait désormais en contact direct avec le monde mal connu de la steppe et du pré-désert. 
8 Ainsi s'expliquent les reconnaissances en force engagées dans ces espaces incontrôlés, dont l'expédition de Cornélius Balbus (en 20-19 av. J.-C.) est une bonne illustration. Il s'agit déjà d'un raid de représailles contre les Gétules et leurs alliés Garamantes; l'analyse par J. Desanges des toponymes transmis par Pline l'Ancien dans sa relation du triomphe du proconsul, permet de conclure que les colonnes romaines s'étaient déployées sur deux axes distincts: l'un à partir de la côte à Sabratha à travers la "Phazanie » - c'est-à-dire les confins tuniso-tripolitains actuels - en direction de la capitale des Garamantes, l'autre en direction du Hodna et le piémont de l'AurèsNemencha. On peut constater que ces deux axes dessinaient, deux siècles à l'avance, les lignes maîtresses de l'expansion romaine future dans l'Afrique présaharienne. Une caractéristique essentielle des peuples auxquels l'armée romaine se trouvait alors confrontée était leur aptitude à nouer à partir d'un réseau étendu de relations coutumières fondées sur le semi-nomadisme pastoral ou le commerce caravanier, de vastes coalitions tribales. Il en résultait une forme d'organisation relationnelle en réseau liée à la fois à la structure tribale de ces peuples et à leurs déplacements sur des axes de communication traditionnels à travers ces marges désertiques. Par le jeu des contacts périodiques entre confédérations nomades sur des axes nord-sud, sur les estives du Tell ou dans les zones d'hivernage du Sahara, ou encore le long d'axes caravaniers traditionnels orientés d'est en ouest comme celui dont le tarif douanier de Zaraï révèlera plus tard la permanence, peut s'expliquer l'étendue géographique des soulèvements berbères contre l'occupant romain qui se propageaient comme par une réaction en chaîne d'un bout à l'autre de la Gétulie avec la complicité d'éléments maures et numides à l'ouest ainsi que celle d'authentiques sahariens du Grand Sud, les Garamantes du Fezzan. Cet effet de "connectivité » et la mobilité extrême de ces cavaliers berbères avait tout pour surprendre leurs adversaires. C'est bien un tel scénario que reproduit sous le règne de Tibère la guerre de Tacfarinas qui constitue pour nous l'épisode le plus connu des réactions africaines à la conquête romaine, grâce au récit qu'en a fait Tacite (Ann., I, 52-53 ; III, 20-21). Elle faisait suite à d'autres guerres Gétules qui avaient eu lieu sous le règne d'Auguste, comme celle conduite en 5-6 de notre ère par Cornelius Cossus qui reçut les honneurs triomphaux avec le surnom de Gaetulicus. Mais cette fois-ci le soulèvement met en scène des peuples très divers, car Tacfarinas ancien auxiliaire de l'armée romaine, avait réussi à entraîner dans sa guerre une partie des Maures voisins des Musulames et les Cinithii qui, par leur localisation dans la Petite Syrte, menaçaient les communications romaines avec les villes de Tripolitaine. Aussi le théâtre des opérations finit-il par s'étendre depuis les hautes plaines axées sur l'Oued Mellègue, domaine des Musulames, jusqu'à la Tripolitaine intérieure parcourue par les Garamantes. La cause de cette conflagration aurait été la création, trois ans avant le début des événements, de la route stratégique qui reliait aux rives de la Petite Syrte le camp de la III ${ }^{\mathrm{e}}$ Légion installé depuis peu à Ammaedara en pays musulame. Une partie de cette voie entre Capsa et Tacape est bien connue par les milliaires de L. Nonius Asprenas datés de 14 ap. J.-C. Cet axe routier où patrouillait l'armée romaine pouvait entraver la liberté de mouvement des populations locales; la guerre de Tacfarinas aurait donc été à l'origine, selon J.-M. Lassère, "un conflit routier ». Une autre explication possible a été proposée par J.-P. Laporte et A M'Charek (EB, XXXII, p. 5152): il s'agit des empiétements opérés par les militaires et autres propriétaires romains qui perturbaient l'économie des Musulames semi-nomades en les privant de leurs meilleures terres de culture. 
9 La guerre de Tacfarinas marquerait bien un tournant essentiel dans l'occupation romaine de la région comme le suggère la conclusion qui lui est donnée, en 29-30, sous le règne de Tibère, par le Proconsul d'Afrique $\mathrm{C}$. Vibius Marsus avec le concours des arpenteurs de la III Légion. Il s'agit de l'opération de cadastration lancée à partir du camp d'Ammaedara et connue par les bornes découvertes dans le sud-est tunisien, au Bled Segui et dans les environs de Gabès. Cette centuriation de grande envergure constituait, en fait, le préalable à une réorganisation ultérieure des confins de la province, car elle servira de base et de référence cartographique aux opérations de délimitation entre les territoires de tribus et de cités effectuées au cours de la période suivante, sous le règne de Trajan tout particulièrement. Un autre tournant décisif se situe effectivement à cette époque quand on passe sur ces confins de l'Empire d'un système "hégémonique » ponctué d'interventions militaires épisodiques de représailles contre les incursions nomades à une forme de contrôle territorial marquée par une occupation militaire permanente de la zone frontière ainsi que par une urbanisation et une romanisation progressive des communautés berbères qui s'y trouvaient incluses.

\section{Nomadisme et frontière romaine : les « trois cercles » (Y. Modéran)}

10 Si l'on examine à présent quel type de relations pouvait s'établir deux siècles après les premiers contacts entre l'armée ou l'administration romaine et les populations nomades (ou semi-nomades) en Afrique du Nord, on retrouve la répartition en dégradé de celles-ci suggérée déjà par Salluste mais répartie à présent par les historiens en «trois cercles» selon la formule proposée par Yves Modéran pour l'antiquité tardive. Une telle répartition avait pris tout son sens dès lors que les frontières de l'Empire s'étaient inscrites dans l'espace du pré-désert en atteignant leur plus grande expansion à l'époque sévèrienne, au début $\mathrm{du} \mathrm{III}^{\mathrm{e}}$ siècle de notre ère, grâce à l'activité déployée tout au long de la frontière d'Afrique par le légat propréteur de la III ${ }^{\mathrm{e}}$ Légion Auguste, C. Anicius Faustus, à partir du quartier général de Lambèse. Une nouvelle série d'avantpostes ont été crées dans le pré-désert comme celui de Messad* dans l'Atlas saharien ou de Bu-Njem* dans l'arrière-pays de la Syrte, pour surveiller les pistes caravanières et les échanges commerciaux dont l'autorité romaine prend aussi le contrôle.

11 Un premier cercle de peuples nomades restés largement en dehors des frontières romaines mais soumis de loin à un tel contrôle à partir de ces antennes sahariennes du limes, englobait des communautés berbères éparses depuis les Pharusiens* du sud marocain jusqu'aux Nasamons et aux Amonniens du désert de Cyrénaïque. Les Pharusii que J. Desanges situe sur le versant saharien du Haut-Atlas sont considérés par Pline comme des Éthiopiens; si l'on en croit Strabon (XVII, 3,7), ils se rendaient parfois au marché de Cirta par un pays marécageux (les Chotts du Sud Oranais selon J. Desanges), ce qui suppose un parcours de près de $900 \mathrm{~km}$. On peut supposer qu'une des fonctions de la cavalerie romaine du poste de Messad (Castellum Dimmidi) placé sur un passage de l'Atlas Saharien devait être de surveiller de tels déplacements au long cours. Parmi les autres peuples restés plus à l'est en marge des frontières romaines, dans les oasis du 
Fezzan, on retrouve les Garamantes, désormais pacifiés si l'on se réfère aux ostraca de $\mathrm{Bu}$ Njem (Gholaia) qui décrivent ces nomades dans le troisième quart du $\mathrm{III}^{\mathrm{e}}$ siècle conduisant tranquillement des ânes ou apportant de l'orge, accompagnés de détachements romains. Or le camp de Bu Njem se trouvait à la limite du pays des Maces sédentarisés. Plus à l'est, les Nasamons sont des nomades plus irréductibles souvent cités jusqu'au $\mathrm{III}^{\mathrm{e}}$ siècle et comme les Garamantes, inscrits pour longtemps au répertoire poétique de la barbarie africaine. Ils se déplaçaient, au gré des saisons des côtes orientales de la Syrte jusqu'à l'oasis d'Augila où ils avaient leur centre. Vers le sud-ouest, ils étaient voisins des Garamantes et vers le nord-ouest des Maces. À basse époque, ils seront remplacés dans les mêmes régions par d'autres nomades redoutables, les Austuriani et les Laguatan qui menaceront les villes de Tripolitaine avant de pénétrer en force, aux époques vandale et byzantine, en Byzacène où ils trouveront des alliés dans les tribus "maures» entrées en dissidence. Selon une hypothèse de Y. Modéran, ces Austurani et Laguatan seraient les héritiers des Nasamons, qui, suite à leur défaite contre les Romains sous Domitien, auraient cédé la prééminence à des fractions devenues dominantes par une restructuration tribale.

Un second cercle de populations, progressivement mis en tutelle par l'administration romaine correspond à des groupes de tribus cantonnées dans la zone-frontière du limes, et avec lesquels, pour les Romains des cités de l'intérieur, les relations étaient plus fréquentes et plus pacifiques. Le champ géographique de cette zone de confins s'élargissait en profondeur d'ouest en est, par une série de décrochements successifs vers le sud: de l'axe routier de la nova praetentura en bordure de la Maurétanie césarienne, il passait par le piémont saharien des Aurès-Nemencha en bordure de la Numidie et par les oasis du Djérid, jusqu'au pré-désert de Tripolitaine, dans le Nefzaoua* et en arrière de la Syrte. Dans cette zone est parachevée la mise en place commencée sous les règnes de Trajan et d'Hadrien, d'une organisation militaroadministrative en réseau, étroitement calquée sur les réalités humaines qu'elle avait pour mission de contrôler : dans ces confins sahariens le limes est articulé autour de pistes stratégiques - pénétrantes ou radiales comme la nova praetentura - permettant la circulation des patrouilles et facilitant les échanges commerciaux à longue distance ; il s'appuie sur une hiérarchie de camps et de postes fixes ou d'avant-postes où sont stationnés des détachements de la III $^{\mathrm{e}}$ Légion ou d'Auxiliaires placés sous le commandement du Légat de Lambèse. Des ouvrages linéaires (fossatum ou clausurae) complétaient, on le verra, ce dispositif de surveillance des déplacements en direction des régions intérieures de l'Empire.

Un exemple des plus représentatifs de ces peuples Gétules inclus dans le réseau militaire romain est celui des Maces dont le pays, entourant les cités du littoral, allait de la Tripolitaine jusqu'au fond de la Grande Syrte; il s'étendait largement dans les grands ouadis au revers du plateau libyen, le Soffegin et le Zemzem, où les recherches archéologiques britanniques ont révélé l'ampleur du processus de sédentarisation à partir du $\mathrm{I}^{\mathrm{er}}$ siècle de notre ère. Un semis très dense d'installations rurales - fortifiées à partir du III siècle - et surtout l'originalité des techniques d'irrigation sont les caractéristiques de cette partie du pré-désert "romano-libyen" vouée à un type d'économie agro-pastorale. Plus à l'ouest sur le revers de l'arc montagneux du sud tunisien, on retrouve les vestiges d'un même type d'aménagement en terrasses dans les vallées, comparables aux jessour actuels des Matmatas* et du Djebel Demmer. Mais on a tout lieu de penser que la vie nomade restait prépondérante dans ce vaste glacis présaharien. Plusieurs groupes tribaux de ce « second cercle» sont localisables en effet 
près des postes ou des avant-postes du limes Tripolitanus qui avaient pour fonction de surveiller leurs déplacements et de prévenir les conflits aux abords des puits dans les zones de transhumance. Les uns sont mentionnés par les auteurs anciens comme les Astahkoures et les Akhemenes ou les Tidamensii (Ptolémée, IV, 3, 6) et les Phazanii (Pline, $\mathrm{V}, 35$ ); les autres par les documents épigraphiques comme pour les Nybgenii* et les Arzuges* dont les noms figurent sur des bornes posées sous le règne de Trajan pour délimiter leurs territoires. Ces deux peuples qui occupaient la région du Nefzaoua, entre les Chotts, le plateau du Dahar et les dunes de l'Erg sont très représentatifs des modalités d'évolution possibles de ces populations nomades au contact des Romains dans la zone frontière du limes. Alors que les Nybgenii étaient centrés dans la partie septentrionale où ils voisinaient le long de la chaîne du Chareb et dans le seuil du Tebaga avec les cités de Capsa et de Tacape, les Arzuges devaient occuper la partie sud du Nefzaoua, au-delà de Bir Soltane dans la direction de Douz. Les premiers sont entrés dans la mouvance du pouvoir romain avec le statut de civitas sous Trajan et celui de municipe sous Hadrien pour leur centre de rassemblement, Turris Tamalleni, situé dans les oasis de Telmine et de Mansourah. Il sera une position-clef sur une rocade du futur limes Tripolitanus. Le semis de vestiges d'exploitations rurales très dense découvert sur leur territoire témoigne d'une sédentarisation avancée dès cette époque ; mais celle-ci n'excluait pas des déplacements saisonniers des éleveurs vers les zones d'épandage des oueds ou vers le littoral et le bled Ségui, ce qui expliquerait le dispositif mis en place par l'administration romaine pour contrôler ces passages dans le seuil du Tebaga et dans les montagnes du Chareb. Les Arzuges, en revanche auraient conservé jusque dans l'antiquité tardive toute leur mobilité et leur structure tribale, ce qui explique leur réputation de passeurs attestée dans la correspondance de St. Augustin. Le nom des Arzuges avait été rapproché, non sans raison peut-être, par Courtois de celui des actuels Berbères Marazig, qui avaient pour centre Douz au sud du Chott el-Djerid, mais dont l'aire de transhumance s'étendait largement du nord du Dahar aux confins de l'Erg.

Un troisième cercle est celui des peuples établis dans les provinces en deçà des frontières romaines. Des Hautes Plaines constantinoises au golfe de Gabès, les anciens peuples Gétules plus ou moins regroupés en confédérations tribales comme celle des Musulames ou des Musuni, ont été engagés, de l'époque flavienne à celle de Septime Sévère, dans un processus de promotion urbaine et de romanisation de leurs élites sociales. Le cas des Capsitani* ou des Cinithii sont à ce titre exemplaires ; leurs centres de rattachement étaient situés pour les premiers, à Gafsa, oasis et position-clef - oppidum magnum atque valens selon Salluste - au nord des montagnes du Chareb qui limitent son territoire étendu dans la steppe tunisienne; pour les seconds à Gigthis, site portuaire ancien et important de la petite Syrte au débouché d'un itinéraire commercial en liaison avec Ghadamès. Les Capsitani étaient considérés par Pline $(\mathrm{V}, 30)$ comme un peuple (natio) plutôt qu'une civitas, ce qui lui confère une certaine importance, mais Ptolémée (IV, 6,11) situe Capsa en Libye intérieure, « ce qui semble indiquer que la ville était considérée comme quelque peu en marge de la province, jusqu'à ce que Trajan en fit un municipe » (J. Desanges, EB, XII, p. 1770). Plus tard, d'après la Table de Peutinger (V, 1), la ville était devenue colonie honoraire. Les Cinithii eux aussi considérés comme des nationes dépendaient en partie d'un praefectus gentis*, ce qui semble impliquer un moindre degré d'intégration dans la romanité, mais leur centre de Gightis devenu très prospère sera municipe sous Antonin le Pieux. Plus à l'ouest l'urbanisation s'était développée autour du territoire des Musulames et des Musuni ; les bornages effectués sous Trajan dans la région de Mactar* et d'Haïdra ont fait récemment l'objet d'une 
réinterprétation ( $E B, X X X I I$, p. 5150-5155). Plutôt qu'un refoulement d'une population nomade, le bornage matérialisait la séparation de zones de statuts juridiques différents: territoires appartenant à des Romains latifundiaires, communautés urbaines d'origine musulame et secteurs laissés à des fractions de la gens Musulamiorum restées fidèles à leur mode de vie traditionnel fondé sur l'élevage extensif. L'essentiel est ici de souligner la persistance sur ces territoires de l'ancienne Gétulie d'une circulation saisonnière des hommes et des troupeaux. À l'appui de cette démonstration nous disposons de plusieurs arguments les uns fondés sur la géographie historique $d u$ nomadisme à la lumière de quelques rares documents épigraphiques ; les autres sur une interprétation plus réaliste d'un certain type d'installations frontalières situées précisément à l'interface des second et troisième cercles de populations berbères, en bordure de la zone du limes.

Sur les rapports du nomadisme et de la frontière romaine en Afrique du Nord, le tarif de Zaraï est un document de premier ordre car il est le seul à permettre d'appréhender le nomade dans sa double fonction d'éleveur transhumant et de transporteur caravanier. L'analyse du document révèle la conjonction de deux flux d'inégale importance: un transport de marchandises de luxe comme le garum et les éponges venant de la Syrte par le sud de l'Aurès et assujettis au paiement du droit de douane du portorium; le second concerne les pequaria, c'est-à-dire les troupeaux qui passaient au poste de Zaraï en franchise. On a tout lieu de penser que ceux-ci venaient de régions plus proches par les vallées mettant en communication le bassin du Hodna avec les hautes plaines constantinoises et qu'il s'agissait bien du bétail des semi-nomades se déplaçant vers leurs pâturages d'été ou vers les marchés du Tell, les nundinae* dont plusieurs sont connus dans la région, comme ceux Ain Kerma ou d'Ain Mechria sur le territoire des Suburbures. Une autre preuve de ces déplacements semi-nomades entre le pré-désert et les régions du Tell repose sur les exemples signalés par J. Desanges de certaines duplications d'ethniques d'après les sources anciennes (Pline, $H N, \mathrm{~V}, 333,337$ ). Ainsi en est-il des Suburbures et des Nicives. Ceux-ci sont attestés sous Vespasien près de Tigisi, sur des terres de la confédération cirtéenne; on les retrouve à basse époque autour de N'gaous aux abords du Hodna. À l'hypothèse traditionnelle du refoulement au désert des tribus nomades ou à leur fractionnement, on préfère désormais celle déjà présentée déjà par A. Berthier, d'une double implantation régionale saisonnière de ces transhumants sur leurs estives du Tell et dans leurs quartiers d'hiver du pré-désert. De tels exemples de double implantation sont aussi connus pour les Maces et les Nasamons.

16 Restent à évoquer ces ouvrages linéaires, murs ou fossés mis en place, vraisemblablement au $\mathrm{III}^{\mathrm{e}}$ siècle dans la zone frontière et sur lesquels Baradez avait attiré l'attention dans le Sud algérien, sous le nom de fossatum Africae en référence aux prescriptions du Code Théodosien. Dans le Sud tunisien, des réalisations du même type ont été reconnues comme celle qui ferme, sur $17 \mathrm{~km}$ de long, le couloir naturel conduisant vers l'Arad et Gabès entre les montagnes de Matmata et du Tebaga. D'autres ouvrages, plus discontinus ferment les passages à travers les montagnes du Chareb qui séparent au nord des Chotts, la région du Nefzaoua de celle du Bled Segui. La plus spectaculaire de ces barrières est celle de Bir oum Ali au sud-est de Gafsa (fig. 2). Enfin, on peut voir des clausurae du même type dans les vallées du Djebel Demmer, en direction de la plaine côtière de la Djeffara, à l'ouest du camp de Ras el-Aïn (Talalati) et au sud de Remada. L'une de ces barrières à guichet se trouve dans le défilé de l'oued Zraïa au nom doublement évocateur puisqu'on le retrouve dans celui antique de Zarai : 
la racine ZRY est celle d'un verbe pan-berbère qui exprime l'action de passer et l'idée de transit ${ }^{1}$ (azray en kabyle, cf. Dallet, p. 958). C'est finalement plutôt en termes de surveillance des courants de circulation, comme un instrument d'administration militaire et non comme une fermeture hermétique de la frontière qu'il convient donc d'interpréter ces installations. Placés en des lieux où semi-nomades et transhumants se concentraient avant de s'engager dans les couloirs conduisant vers les aires de culture et d'estivage de la steppe ou de la plaine côtière, ces «barrières " permettaient d'en réguler le flux - et de le protéger contre d'éventuels pillards - en le canalisant vers les principaux passages obligés de la zone frontière. Un tel contrôle pouvait avoir pour but de faire respecter le calendrier des récoltes pour les troupeaux transhumants, mais aussi d'exercer une taxation douanière sur les marchandises transportées vers les marchés périodiques situés dans la zone de contact entre unités naturelles d'économies complémentaires.

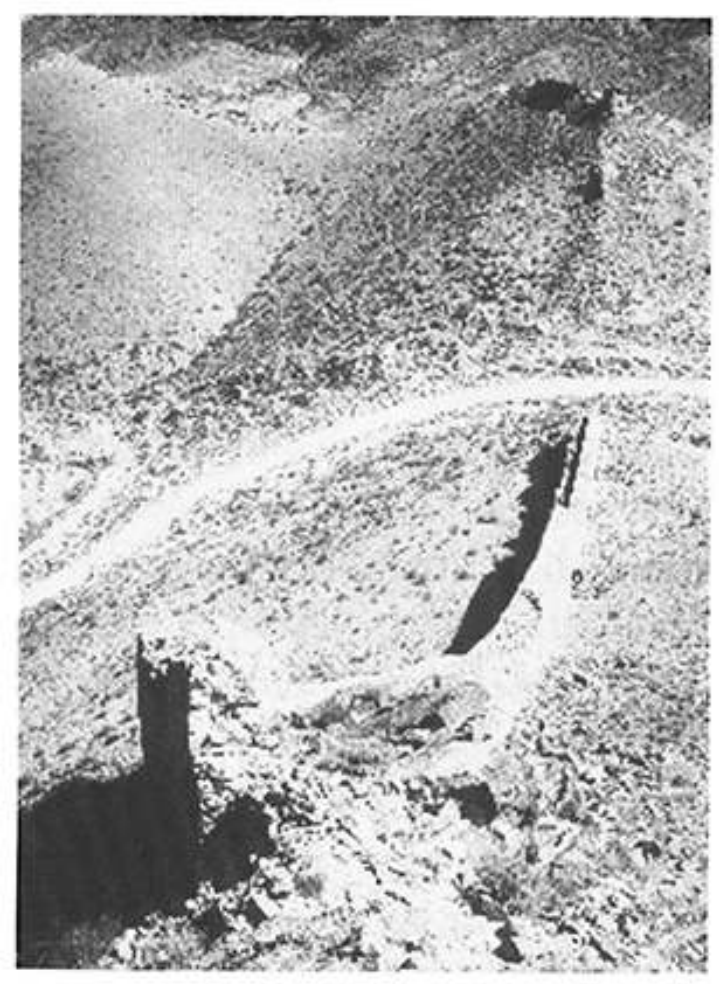

Fig. 2. BARRIÈre DÉFENSIVE DE BIR OUM ALI, SUD-EST DE GAFSA.

Cliché P. Trousset.

\section{BIBLIOGRAPHIE}

BARKER G., GILBERSON D., JONES B., MATTINGLY D., 1996 - Farming the Desert, The UNESCO Libyan Valleys Archaeological Survey, Tripoli.

BERTHIER A., 1981 - La Numidie, Rome et le Maghreb, Paris, Picard. 
CAMPS G., 1960 - « Massinissa ou les débuts de l'Histoire », Libyca, VIII.

CAMPS G., 1985 - « Pour une lecture naïve d'Hérodote, les récits libyens », Storia della Storiografia, 7.

DALLET J.-M., 1982 - Dictionnaire kabyle-français, Paris, SELAF/Peeters.

DAUGE Y.-A., 1981 - Le Barbare, Recherche sur la conception romaine de la barbarie et de la civilisation, Bruxelles, Latomus.

DESANGES J., 1962 - Catalogue des tribus africaines de l'antiquité classique à l'Ouest du Nil, Dakar.

DESANGES J., 1978 - Recherches sur l'activité des Méditerranéens aux confins de l'Afrique (Vie siècle avant J.-C.-IVe siècle après J.-C.), Rome.

MATTINGLY D., 2003 - The Archaeology of Fazzan, vol. 1, Synthesis), Tripoli, Londres.

MODERAN Y., 2003 - Les Maures et l'Afrique romaine (IVe-VII siècle), Rome.

TROUSSET P., 1982 - « L'image du nomade saharien dans l'historiographie antique », dans Production pastorale et société, MSH, Paris.

TROUSSET P., 2005 - « Le tarif de Zaraï : Essai sur les circuits commerciaux dans la zone présaharienne ", Ant afr., t. 38-39, p. 355-373.

\section{NOTES}

1. La forme Zarai elle-même - à restituer en *[zĂray], qui donne en berbère [azĂray, azray] -, s'analyse comme un nom d'action verbal, « le passage, le transit » à partir de la racine ZRY. NDLR.

\section{INDEX}

Mots-clés : Antiquité, Commerce, Economie, Géographie, Sahara 